\title{
Activation of the Unfolded Protein Response Enhances Motor Recovery after Spinal Cord Injury
}

\section{Citation}

Valenzuela, V., E. Collyer, D. Armentano, G. B. Parsons, F. A. Court, and Claudio Hetz. 2012. Activation of the unfolded protein response enhances motor recovery after spinal cord injury. Cell Death \& Disease 3(2): e272.

\section{Published Version}

doi:10.1038/cddis.2012.8

\section{Permanent link}

http://nrs.harvard.edu/urn-3:HUL.InstRepos:8605331

\section{Terms of Use}

This article was downloaded from Harvard University's DASH repository, and is made available under the terms and conditions applicable to Other Posted Material, as set forth at http:// nrs.harvard.edu/urn-3:HUL.InstRepos:dash.current.terms-of-use\#LAA

\section{Share Your Story}

The Harvard community has made this article openly available.

Please share how this access benefits you. Submit a story.

Accessibility 


\title{
Activation of the unfolded protein response enhances motor recovery after spinal cord injury
}

\author{
V Valenzuela ${ }^{1,2,3}$, E Collyer ${ }^{2}$, D Armentano ${ }^{4}$, GB Parsons ${ }^{4}$, FA Court ${ }^{\star 2,5}$ and C Hetz ${ }^{\star 1,3,5,6}$
}

Spinal cord injury (SCl) is a major cause of paralysis, and involves multiple cellular and tissular responses including demyelination, inflammation, cell death and axonal degeneration. Recent evidence suggests that perturbation on the homeostasis of the endoplasmic reticulum (ER) is observed in different $\mathrm{SCl}$ models; however, the functional contribution of this pathway to this pathology is not known. Here we demonstrate that SCl triggers a fast ER stress reaction (1-3 $\mathrm{h}$ ) involving the upregulation of key components of the unfolded protein response (UPR), a process that propagates through the spinal cord. Ablation of X-box-binding protein 1 (XBP1) or activating transcription factor 4 (ATF4) expression, two major UPR transcription factors, leads to a reduced locomotor recovery after experimental SCI. The effects of UPR inactivation were associated with a significant increase in the number of damaged axons and reduced amount of oligodendrocytes surrounding the injury zone. In addition, altered microglial activation and pro-inflammatory cytokine expression were observed in ATF4 deficient mice after SCl. Local expression of active XBP1 into the spinal cord using adeno-associated viruses enhanced locomotor recovery after SCl, and was associated with an increased number of oligodendrocytes. Altogether, our results demonstrate a functional role of the UPR in SCl, offering novel therapeutic targets to treat this invalidating condition.

Cell Death and Disease (2012) 3, e272; doi:10.1038/cddis.2012.8; published online 16 February 2012

Subject Category: Neuroscience

Spinal cord injury $(\mathrm{SCl})$ due to mechanical trauma, ischemia, or tumor invasion leads to invalidating locomotor impairment. $\mathrm{SCl}$ often affects individuals in their productive age, having an enormous social and economic impact. The contribution of the various subcellular compartments to the process of tissue damage triggered by $\mathrm{SCl}$ has not been clearly elucidated. A number of pathological conditions affecting the nervous system, including $\mathrm{SCl}$, can interfere with the function of a specific subcellular organelle, the endoplasmic reticulum (ER), resulting in a cellular condition termed ER stress. ${ }^{1,2}$ To alleviate ER stress, cells activate an integrated signaling pathway known as the unfolded protein response (UPR), which reestablish homeostasis by decreasing the extent of protein misfolding. ${ }^{2}$ Conversely, chronic or irreversible ER stress triggers apoptosis to eliminate nonfunctional cells. ${ }^{3}$

The UPR is initiated by several stress sensors, including inositol-requiring enzyme-1 (IRE1) $\alpha$, which is a kinase and endoribonuclease that upon activation, initiates the splicing of the mRNA encoding the transcriptional factor $\underline{X}$-box-binding protein 1 (XBP1), ${ }^{2,4}$ converting it into a potent activator of multiple UPR-responsive genes (termed XBP1s). XBP1s controls the expression of genes involved in protein folding, secretion, and protein quality control. ${ }^{5}$ IRE $1 \alpha$ also regulates other signaling events including the activation of JNK (c-Jun $\mathrm{N}$-terminal kinases), modulating apoptosis and autophagy levels, in addition to degrading a subset of mRNA through its RNAse activity (reviewed in Hetz $^{2}$ ). Other important UPR effects are mediated by the stress sensor PKR-like endoplasmic reticulum kinase (PERK), which phosphorylates the eukaryotic translation initiation factor $2 \alpha$ (elF2 $\alpha$ ), inhibiting protein translation into the ER, and reducing the overload of misfolded proteins. ${ }^{6}$ elF2 $\alpha$ phosphorylation also triggers the specific translation of activating transcription factor 4 (ATF4), which is essential for the upregulation of different foldases and the regulation of the redox and metabolic status of the cell. ${ }^{7}$ Under prolonged ER stress, ATF4 also controls the expression of pro-apoptotic components such as CCAAT/enhancerbinding protein (C/EBP) (CHOP)/GADD153 and several

\footnotetext{
${ }^{1}$ Biomedical Neuroscience Institute, Faculty of Medicine, University of Chile, Santiago, Chile; ${ }^{2}$ Millennium Nucleus for Regenerative Biology, Faculty of Biology, P. Catholic University of Chile, Santiago, Chile; ${ }^{3}$ Center for Molecular Studies of the Cell, Institute of Biomedical Sciences, University of Chile, Santiago, Chile; ${ }^{4}$ Department of Molecular Biology, Genzyme Corporation, 49 New York Avenue, Framingham, MA 01701, USA; ${ }^{5}$ Neurounion Biomedical Foundation, Santiago, Chile and ${ }^{6}$ Department of Immunology and Infectious diseases, Harvard School of Public Health, Boston, MA, USA

*Corresponding author: C Hetz, Institute of Biomedical Sciences, University of Chile, Independencia 1027, PO BOX 70086, Santiago, Chile. Tel: + 5629786506 ;

Fax: + 562 9786871; E-mail: chetz@med.uchile.cl; Website: http://ecb-icbm.med.uchile.cl/ and Department of Immunology and Infectious Diseases, Harvard School of Public Health, 651 Huntington Av, Boston, MA 02446, USA. E-mail: chetz@hsph.harvard.edu

or FA Court, Department of Physiology, Faculty of Biology, P. Universidad Católica de Chile, Av. B. O'Higgins 340/Casilla 114-D, Santiago 8331150, Chile.

Tel: + 562 6862899; Fax: + 562 3541850; E-mail: fcourt@ bio.puc.cl

Keywords: spinal cord injury; unfolded protein response; endoplasmic reticulum stress; XBP1; motor dysfunction

Abbreviations: ER, endoplasmic reticulum; UPR, unfolded protein response; $\mathrm{SCl}$, spinal cord injury; ATF4, activating transcription factor 4; XBP1, X-box-binding protein 1; XBP1s, spliced XBP1; IRE1, inositol-requiring enzyme-1; PERK, PKR-like endoplasmic reticulum kinase; CHOP, CCAAT/enhancer-binding protein (C/EBP); elF2a, eukaryotic translation initiation factor 2A; BiP, binding immunoglobulin protein; BMS, Basso Mouse Scale; JNK, c-Jun N-terminal kinases; AAV, Adeno-associated virus; GFP, green fluorescent protein; GFAP, glial fibrillary acidic protein; NeuN, neuronal nuclei; Olig2, oligodendrocyte transcription factor 2; Cd11b, cluster of differentiation $11 \mathrm{~b}$

Received 04.1.12; accepted 05.1.12; Edited by A Verkhratsky
} 
BCL-2 family members, including BCL-2, BIM, and PUMA, among others. ${ }^{3}$

Correlative studies indicate that markers of ER stress are observed in different models of $\mathrm{SCl}$ triggered by trauma (by contusion and hemisection) and ischemia. ${ }^{8-12}$ Using a contusion model, ER stress responses were observed in the damaged zone, including XBP1 mRNA splicing, in addition to the induction of $\mathrm{CHOP}$ and the ER chaperone BiP. ${ }^{10}$ Neuronal cells presented a fast and transient activation of UPR markers, whereas oligodendrocytes and astroglia showed sustained activation for several days. ${ }^{13}$ Interestingly, signs of ER stress were also detected at some distance from the injury site, ${ }^{8}$ suggesting a spread of the pathological stress process. Similarly, damage to the spinal cord by transient ischemia correlates with increased ER stress levels. ${ }^{12,14} \mathrm{~A}$ recent study indicated that deletion of chop enhances motor recovery after moderate levels of $\mathrm{SCl}$ induced by contusion, ${ }^{13}$ whereas CHOP deficiency did not have any effect under conditions of severe $\mathrm{SCl} .{ }^{15}$ As $\mathrm{CHOP}$ expression is one of dozens downstream targets of the UPR, ${ }^{5}$ and the ATF4/CHOP pathway is activated by a variety on non-ER stress-related stimuli including DNA damage, ${ }^{16}$ oxidative stress, ${ }^{17}$ metabolic changes, ${ }^{18}$ inflammation, among other effects, ${ }^{19}$ direct manipulation of proximal UPR components is required to directly define the contribution of ER stress to $\mathrm{SCl}$.

In this study, we confirm a massive and early upregulation of key ER stress markers in the injury zone after spinal cord hemisection, including a rapid activation of XBP1 and ATF4, in addition to other important UPR downstream target genes. To address the possible impact of ER stress in the functional recovery after experimental $\mathrm{SCl}$, we analyzed the susceptibility of ATF4- and XBP1-deficient mice to experimental SCI. Remarkably, both mouse models presented significant impairment of locomotor recovery after $\mathrm{SCl}$ compared with wild-type animals. These effects were associated with drastic changes at the level of oligodendrocyte number, microglia activation, inflammation markers, and axonal degeneration. Furthermore, treatment of wild-type mice with Adeno-associated viruses (AAV2) to deliver an active from of XBP1 locally into the spinal cord enhanced fine locomotor movements and increased oligodendrocyte content in the injury zone. Overall, our results indicate a detrimental impact of ER stress in $\mathrm{SCl}$, identifying an unanticipated signaling target to treat this pathological condition affecting motor and sensory function.

\section{Results}

Early and sustained activation of the UPR after spinal cord hemisection. To monitor the occurrence of ER stress after $\mathrm{SCl}$, we first validated the possible activation of UPR components in wild-type mice after spinal cord hemisection. A significant and progressive upregulation of XBP1 mRNA splicing was detected by RT-PCR $6 \mathrm{~h}$ after $\mathrm{SCl}$ in the injured region, and this change was sustained for several days (Figure 1a). We also observed a significant increase in XBP1 mRNA splicing in rostral regions far from the injury zone a
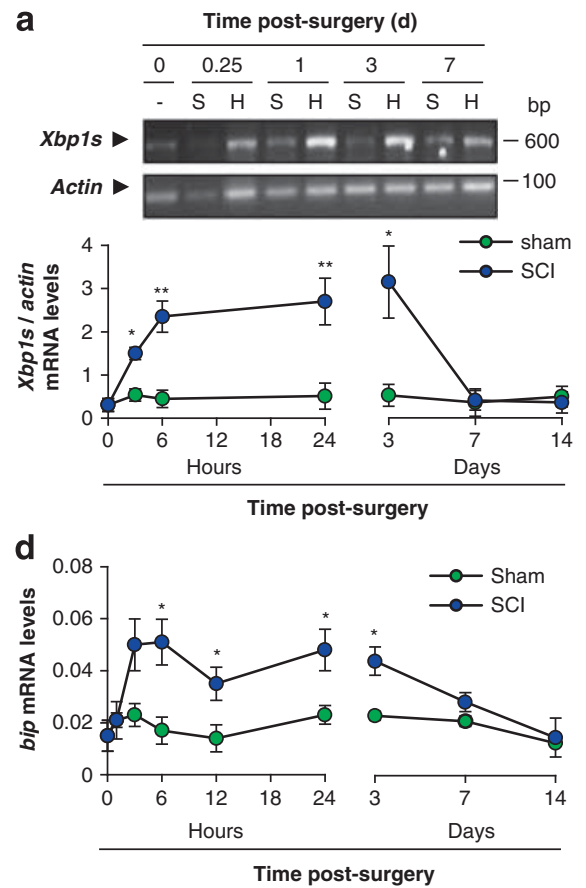

b
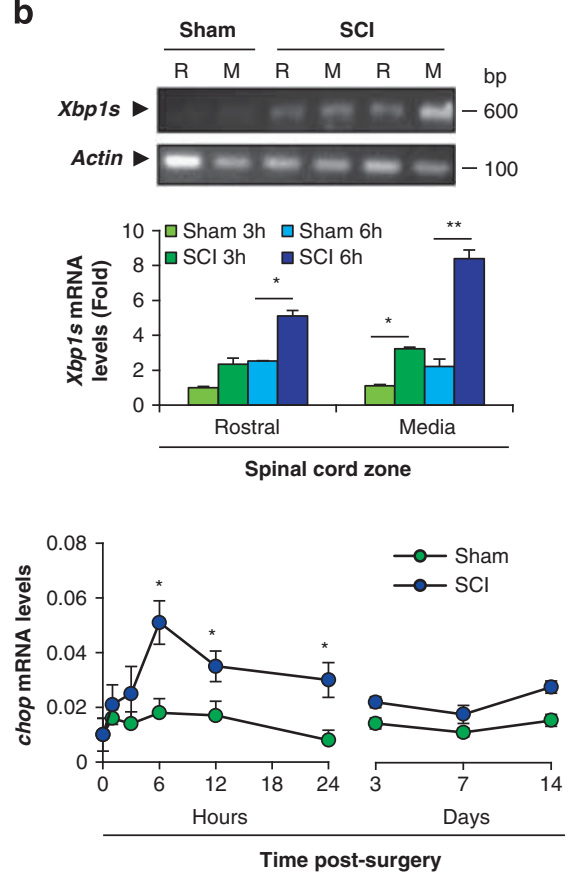

C
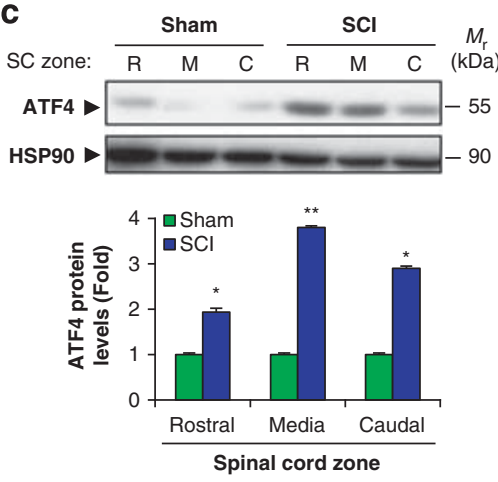

e

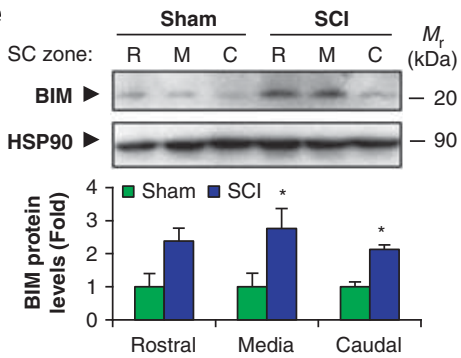

Figure 1 UPR activation after spinal cord hemisection. (a) Wild-type mice were spinal cord-hemisected (H) or sham-operated (S) at the T12 vertebral level. 0.25 ( 6 h), 1, 3, 7, and 14 days after the surgical procedure, tissue from the operated region of the spinal cord was extracted and processed to measure spliced Xbp1 (Xbp1s) mRNA levels by RT-PCR. Actin mRNA was used for normalization. (b) The same procedure was used to study Xbp1 mRNA splicing at 3 and $6 \mathrm{~h}$ after SCl at the injured region (media) and at a distance from the injury site (rostral) (lower panel). Xbp1s levels were quantified and normalized using actin mRNA levels. (c) ATF4 protein levels were measured by western blot and semiquantified by normalizing with HSP90 protein levels. Blot and graph at $6 \mathrm{~h}$ after sham or hemisection from rostral (R), operated (M) and caudal (C) regions are shown. (d) bip and chop mRNA levels were quantified at the indicated times after damage by real-time PCR. (e) BIM protein levels at $6 \mathrm{~h}$ after $\mathrm{SCl}$ were measured by western blot. Protein levels were normalized with HSP90 levels and then normalized to the sham-operated condition. Mean \pm S.E.M. ${ }^{*} P<0.05$; ${ }^{* *} P<0.005$; Student's $t$-test; $n=3$ animals per group for protein and mRNA analysis 
(Figure 1b). Similarly, we found a significant increase in ATF4 protein levels $6 \mathrm{~h}$ after $\mathrm{SCl}$ in the injury site, in addition to distant regions of the spinal cord (Figure 1c). These changes were observed as early as $1 \mathrm{~h}$ after $\mathrm{SCl}$ (Supplementary Figure S1A). In agreement with these findings, we detected a sustained upregulation of classical UPR-target genes after $\mathrm{SCl}$, including bip and chop (Figure 1d). In addition, we detected the upregulation of additional markers including BIM (Figure 1e), a pro-apoptotic BCL-2 family member modulated by $E R$ stress, ${ }^{20}$ and increased accumulation of polyubiquitinated proteins (Supplementary Figure S1B) after $\mathrm{SCl}$. In summary, we were able to confirm previous findings indicating an early and sustained activation of the UPR after $\mathrm{SCl}$, reinforcing the idea that protein-folding stress is a major cellular reaction under this condition.

ATF4 and XBP1 deficiency attenuates locomotor recovery after $\mathbf{S C l}$. Activation of the UPR has two paradoxical consequences, adaptation to stress, improving cell survival, or the induction of apoptosis programs to eliminate the chronically damaged cell. To determine the possible functional role of proximal UPR signaling components in locomotor recovery after $\mathrm{SCl}$, we subjected ATF4 knockout mice (atf4 ${ }^{-1-}$ ) and littermate controls to spinal cord hemisection. Analysis of locomotor activity using the Basso Mouse Scale (BMS) open field test ${ }^{21}$ revealed a delayed and reduced recovery of atf $4^{-1}$ mice when compared with hemisected wild-type mice (Figure 2a left panel). A similar result was obtained in the BMS subscore (Figure 2 a right panel), which can detect differences in the fine details of locomotion that may not be apparent in the overall BMS score. ${ }^{21}$ No differences in locomotor capacity were observed between non-injured control and atf $4^{-/-}$mice by BMS or by monitoring rotarod performance (Figure 2a and
Supplementary Figure S2A), indicating that basal locomotor capacity is not compromised in atf4 ${ }^{-1-}$ mice.

Under ER stress conditions, ATF4 and XBP1 have distinct effects on ER homeostasis because of the transcriptional regulation of differential subsets of target genes. ${ }^{22}$ As ATF4 is also activated by many processes that are not related to ER stress, ${ }^{19}$ we decided to further analyze the possible contribution of the UPR in $\mathrm{SCl}$ with a direct genetic manipulation. We evaluated the role of XBP1, which is one of the key components of the UPR and represents the most conserved ER stress signaling branch in evolution. ${ }^{2}$ We recently generated a viable conditional knockout mouse model to delete $x b p 1$ in the nervous system using the Nestin promoter to express Cre $\left(\mathrm{XBP}^{\mathrm{Nes}-{ }^{-}}\right)$, which do not present basal motor phenotypes. ${ }^{23}$ Nestin-Cre system is predicted to induce deletion of oligodendrocytes and astrocytes, in addition to neurons. $^{24}$ Remarkably, XBP1 ${ }^{\mathrm{Nes}-/-}$ animals showed a marked reduction in locomotor recovery after $\mathrm{SCl}$ by both the BMS score and subscore analysis (Figure $2 \mathrm{~b}$ ). Together, the side-by-side comparison of these two independent UPR knockout mice revealed a clear functional role for ER stress in $\mathrm{SCl}$.

Enhanced axonal degeneration and altered cellular environment in ATF4-deficient mice after $\mathbf{S C l}$. We then evaluated the impact of ATF4 deficiency on the cellular alterations classically associated with $\mathrm{SCl}$. In agreement with the negative impact of ATF4 deficiency on locomotor recovery after $\mathrm{SCl}$, atf4 ${ }^{-1-}$ mice presented fewer axons with normal morphology after $\mathrm{SCl}$ compared with control mice, as measured $2 \mathrm{~mm}$ caudal to the injury zone in toluidine blue-stained tissue (Figure 3a). No differences were observed in axonal density in atf4 ${ }^{-/}$on the contralateral, non-injured side (Figure 3a). As a control, a

$$
\begin{aligned}
& \text {..e.- atf } 4^{+/+} \text {Sham } \\
& \text {-.- atf } 4^{-1} \text { Sham } \\
& \text {-0- } \mathrm{atf}^{+/+} \mathrm{SCl} \\
& -a^{-1 f 4^{-}}{ }^{-\mathrm{SCl}}
\end{aligned}
$$

b

$$
\begin{aligned}
& \text {-.- XBP1 }{ }^{\mathrm{WT}} \text { Sham } \\
& \text {-.-XBP1 }{ }^{\text {Nes-l- }} \text { Sham } \\
& \multimap \mathrm{XBP}^{\mathrm{WT}} \mathrm{SCl} \\
& \longrightarrow \mathrm{XBP}^{\text {Nes-l }} \mathrm{SCl}
\end{aligned}
$$
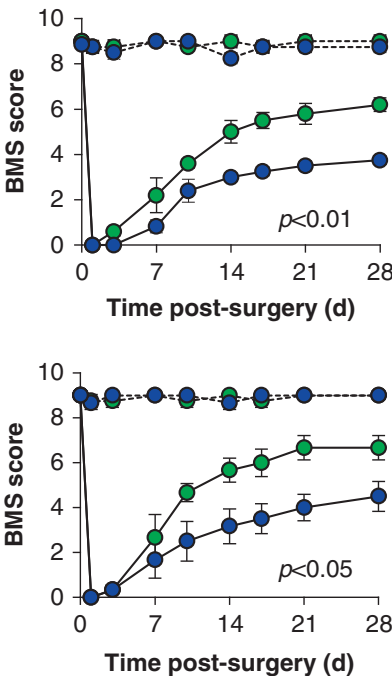
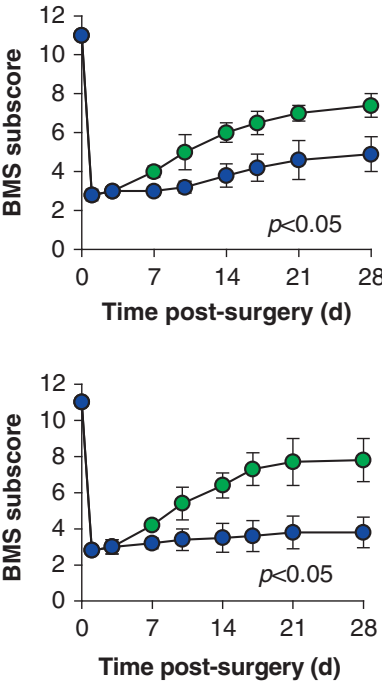

Figure 2 Role for UPR in locomotor recovery after SCl. (a) atf4 ${ }^{+/+}$and atf4 ${ }^{-/}$mice were hemisected at the $\mathrm{T} 12$ vertebral level. Their locomotion recovery pattern was monitored before ( 0 day) and after spinal cord hemisection using the BMS open-field test to determine their locomotor capabilities (left plot). The BMS subscore was quantified to assess locomotor recovery of finer movements (right plot). (b) The same as in (a), but comparing XBP $1^{\text {WT }}$ with XBP ${ }^{\text {Nes }-l-}$ mice. Mean \pm S.E.M. Statistical differences were analyzed by a two-way repeated-measures ANOVA followed by Bonferroni's post hoc test; $P$-values for $\mathrm{SCl}$ group comparison are indicated in the graph; $n=8$ animals per group 
a

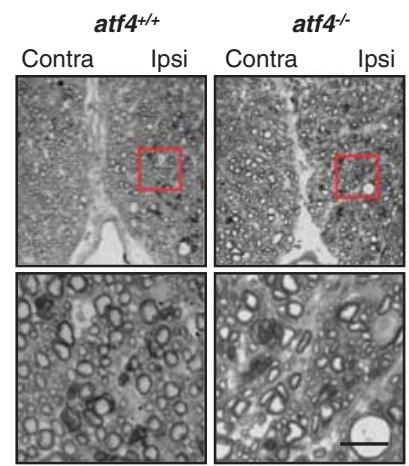

C
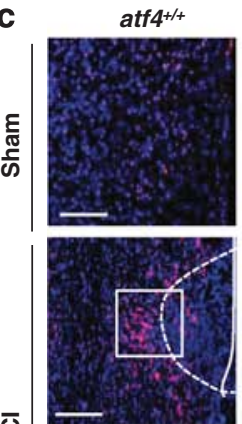

ভ

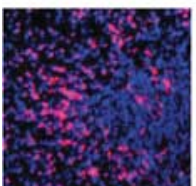

atf4-
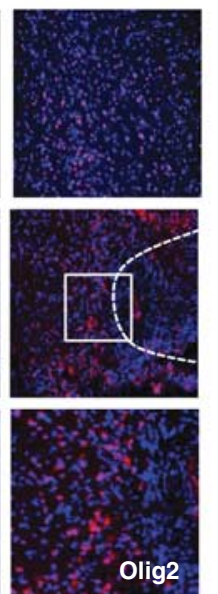

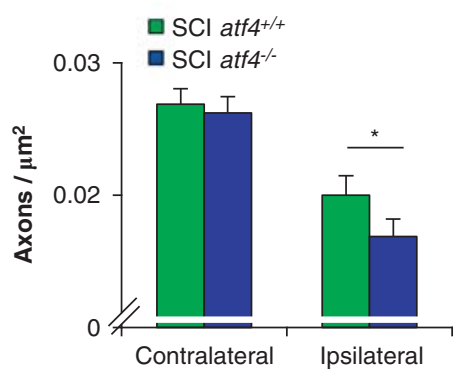

b

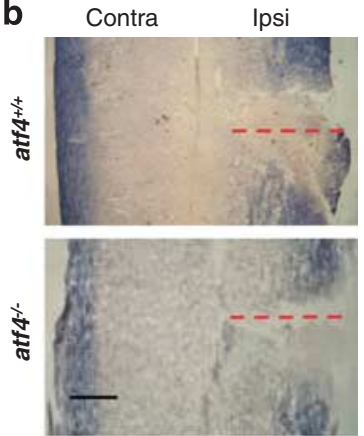

d
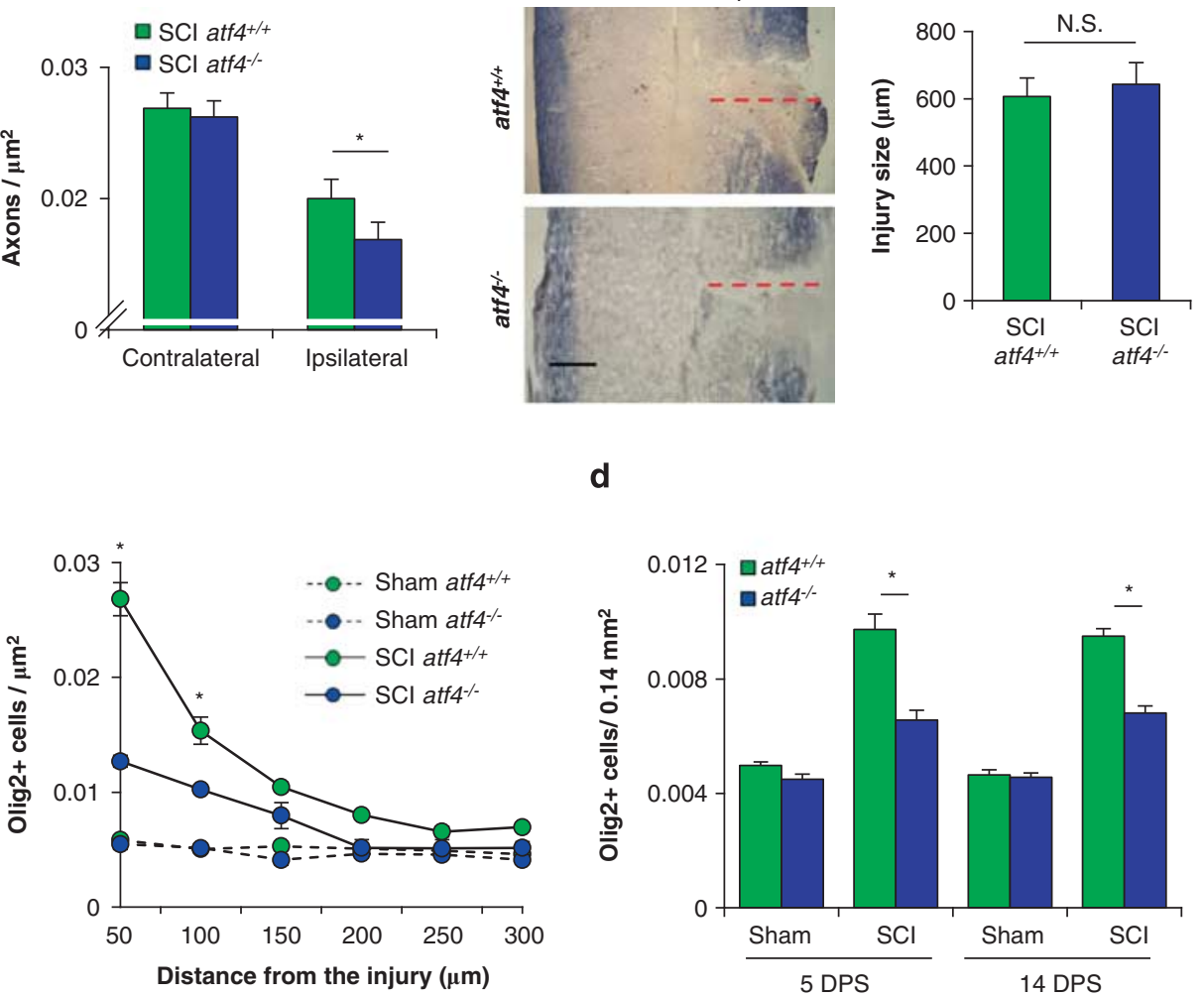

Figure 3 Altered cellular environment in ATF4-deficient mice after SCl. (a) atf4 ${ }^{+/+}$and atf4 ${ }^{-/}$mice were spinal cord-hemisected or sham-operated at the T12 vertebral level. At 14 days after surgery, spinal cord tissue was stained with toluidine blue to study axonal integrity (left panel). Intact axons from corticospinal tracts were quantified and expressed as axonal density values (right panel). (b) Spinal cord tissue from hemisected atf4 ${ }^{+/+}$and atf4 ${ }^{-/}$mice were longitudinally sectioned to analyze the injury size by eriochrome/cyanine myelin staining. Transversal injury length was measured from the edge of the spinal cord to the midline as shown by the red-dotted line. (c) atf $4^{+/+}$and atf $^{-1-}$ mice were spinal cord-hemisected or sham-operated at the T12 vertebral level. At 5 days after surgery, spinal cord tissue processed for immunofluorescence for Olig2, to study oligodendrocytes and its progenitors (red); nuclei were counterstained using Hoechst (blue). Olig2-positive particles co-localizing with Hoechst were quantified every $50 \mu \mathrm{m}$ starting at the injury site. (d) The same procedure as (c), but total Olig2/Hoechst-positive particles were quantified in a semicircular area of $300 \mu \mathrm{m}$ radius surrounding the injury zone at 5 and 14 days post-surgery (DPS). Mean \pm S.E.M. ${ }^{*} P<0.05$; Student's $t$-test; $n=3$ animals per group. Scale bars, $20 \mu \mathrm{m}$ in (a), $200 \mu \mathrm{m}$ in (b), and $100 \mu \mathrm{m}$ in (c). Bars indicate S.E.M.

we performed eriochrome/cyanine staining to monitor the extent of the mechanical damage in both wild-type and $a t f 4^{-/}$mice, which was virtually identical (Figure $3 \mathrm{~b}$ ), also indicating that the surgery protocol was highly reproducible.

In addition to axonal degeneration, many different cellular processes are triggered by $\mathrm{SCl}$, and involve multiple cell types. To define the impact of the UPR on the cellular environment in the injury zone of atf $4^{-/-}$mice, we monitored glial reactions 5 and 14 days after surgery, at the beginning of the locomotor recovery phase according to the BMS score (Figure 2). Increased accumulation of oligodendrocytes was observed in the area surrounding the injury zone of wild-type mice, as monitored by counting the number of olig2-positive cells by immunofluorescence (Figures $3 \mathrm{c}$ and $\mathrm{d}$ and Supplementary Figure S2B). This increase was ablated in $\mathrm{atf}^{-/-}$mice (Figure 3c). No significant differences were observed in the total number of neurons in the injury area by neuronal nuclei (NeuN) staining in either control or atf4 ${ }^{-/-}$mice after 14 days of spinal cord hemisection (Supplementary Figure S2C).

We also monitored the reaction of microglia and astrocytes after genetically targeting ATF4. Activation of microglia was assessed using $\mathrm{Cd11b}$ staining. The classical microglial reaction observed after $\mathrm{SCl}$ was drastically reduced in atf $^{-1-}$ mice 5 days after SCI (Figure 4a). In sharp contrast, analysis of astrocyte distribution by glial fibrillary acidic protein (GFAP) immunostaining did not reveal differences between control and atf $4^{-1-}$ mice at 5 or 14 days after $\mathrm{SCl}$ (Figure 4b and Supplementary Figure S2D). Cell density was assessed by quantifying the number of cell nuclei after Hoechst staining, which possibly represents cellular infiltration and proliferation of macrophages. This measurement was also enhanced in atf4 ${ }^{-1-}$ mice after SCI (Supplementary Figure S2E). We also monitored the levels of several proinflammatory cytokines that are induced upon $\mathrm{SCl}$ including IL- $1 \beta$, TNF $\alpha$ and IL-6. Real-time PCR analysis revealed an induction of these three cytokines in the spinal cord area 
a

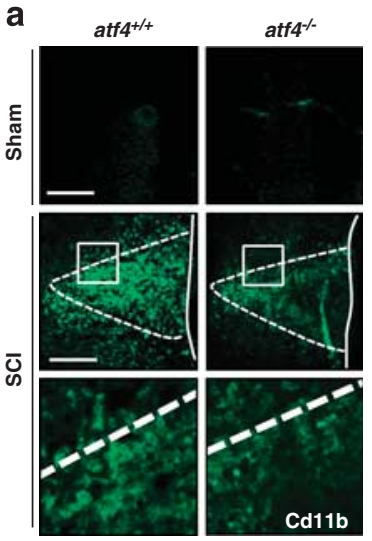

b

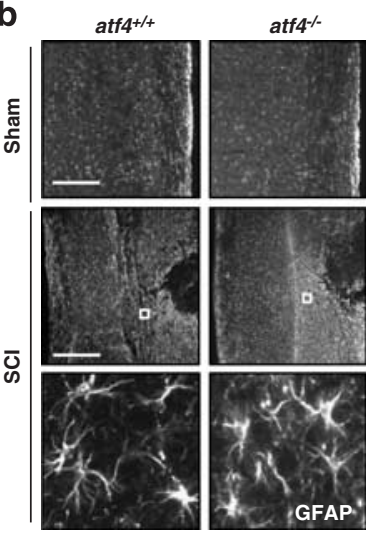

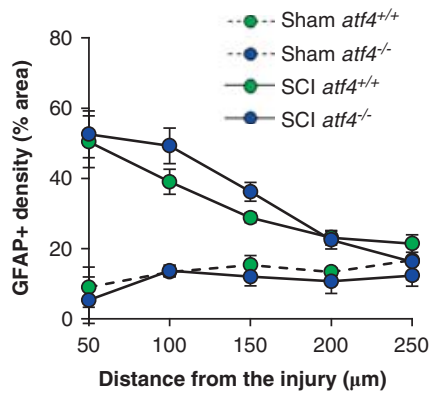
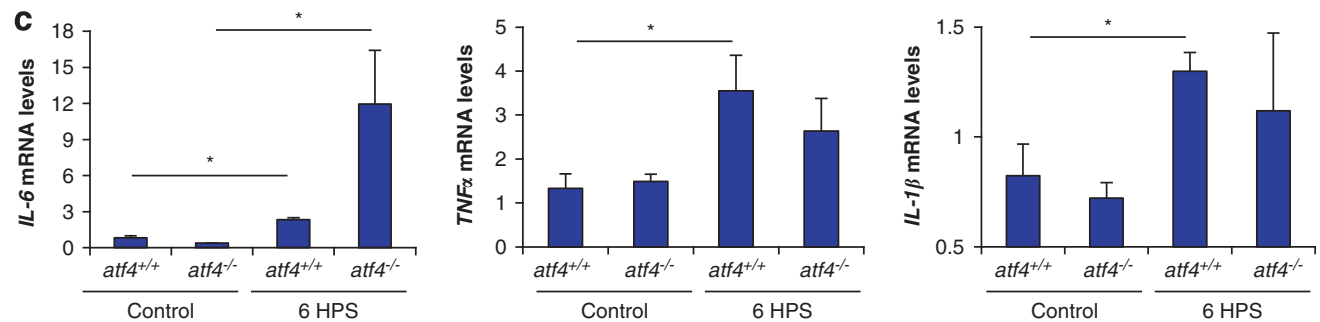

Figure 4 Altered inflammatory response in UPR-deficient mice after SCl. (a) atf4 ${ }^{+/+}$and atf4 ${ }^{-/}$mice were spinal cord-hemisected or sham-operated at the T12 vertebral level. At 5 days after surgery, spinal cord tissue was extracted and processed for immunofluorescence for the microglial marker Cd11b at the injury zone (left panel). The density for Cd11b staining was quantified at the injured zone delimited with a dotted line (right plot) at 5 and 14 days post-surgery (PDS). (b) Activated astrocytes were studied by GFAP immunostaining. GFAP-positive label surrounding the injury zone was quantified every $50 \mu \mathrm{m}$ from the injury (right panel). (c) Inflammatory cytokines were analyzed from injured spinal cord tissue of atf $4^{+/+}$and atf ${ }^{-/}$mice by real-time PCR $6 \mathrm{~h}$ after surgery (HPS). Mean \pm S.E.M. ${ }^{*} P<0.05 ;{ }^{* \star} P<0.005 ;$ Student's $t$-test; $n=3$ animals per group. Scale bars, $300 \mu \mathrm{m}$ in (a) and $20 \mu \mathrm{m}$ in (b)

surrounding the injury zone (Figure 4c). A fivefold increase in IL-6 mRNA levels was observed in atf4 ${ }^{-/}$mice $6 \mathrm{~h}$ after hemisection when compared with atf $4^{+/+}$mice (Figure 4c). TNF $\alpha$ levels were slightly decreased in atf4 ${ }^{-/-}$, whereas IL-1 $\beta$ was not altered (Figure 4c). Taken together, our results indicate that ATF4 expression has a broad impact on the cellular and functional alterations observed after SCl.

XBP1s gene transfer into the $\mathrm{SCl}$ site enhances locomotor recovery. Our studies using XBP1-deficient animals indicate that the UPR has a functional role in alleviating ER stress in SCl. To test the possible therapeutic impact of further enhancing UPR responses after SCl, we developed a gain of function approach to artificially activate the UPR. We generated serotype 2 AAV-based vector to locally deliver the spliced form of XBP1 into the damaged area of the spinal cord. The vector was bicistonic and expressed green fluorescent protein (GFP) to monitor efficiency of transduction. We performed spinal cord hemisection and immediately delivered $2 \mu \mathrm{l}\left(10^{12} \mathrm{DRP} / \mathrm{ml}\right)$ of AAV2, expressing either GFP alone or the XBP1s transgene into the injury zone. This strategy leads to a local and partial transduction of cells closely surrounding the injury site (Figure 5a). Although the area transduced by AAV was small, we observed a slight, but significant, improvement of locomotor performance after 28 days of $\mathrm{SCl}$ in animals injected with AAV2-XBP1s when compared with animals injected with the control virus (Figure $5 b$ ). Furthermore, improvement of locomotor performance was evident when the BMS subscore was analyzed, which reveals finer aspects of locomotor function, observing a significant difference in the locomotor recovery of mice injected with XBP1s AAVs between 14 and 35 days after $\mathrm{SCl}$ (Figure $5 \mathrm{c}$ and Supplementary Figure S3a). The late effects of AAV2mediated delivery of XBP1s transgene are consistent with the reported delay of around 7-14 days for gene expression after AAV transduction. ${ }^{25,26}$ Most of the cells transduced with $A A V$ in the spinal cord were neurons and oligodendrocytes, as predicted for the serotype 2 of the viral particles (Figure $5 d$ and Supplementary Figure S3a). Global analysis of cellularity in the injury zone by Hoechst staining indicated a reduction of cellular density after delivering XBP1s-expressing AAVs (Figures $5 \mathrm{e}$ and $\mathrm{g}$ ). In addition, the accumulation of Olig2positive cells was significantly increased in the injury zone of AAV-XBP1s-treated mice when compared with mice injected with control viruses (Figures $5 f$ and $g$ ). In summary, these results suggest that although the pathway is already activated in $\mathrm{SCl}$, forced expression of active XBP1s locally into the spinal cord can still improve locomotor performance after experimental SCl.

\section{Discussion}

$\mathrm{SCl}$ is a major cause of locomotor impairment and paralysis in the world. However, the molecular events underlying the cellular and functional alterations associated with $\mathrm{SCl}$ are not fully understood. Identifying primary signaling events initiated by $\mathrm{SCl}$ is essential for future therapeutic interventions aiming 
a

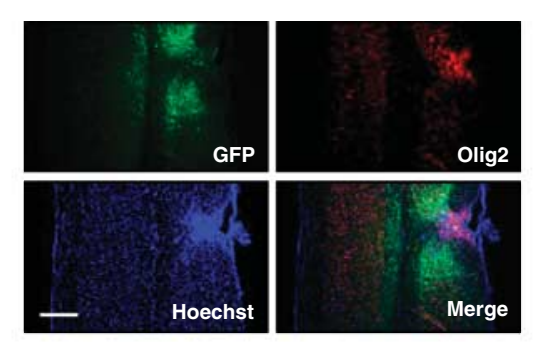

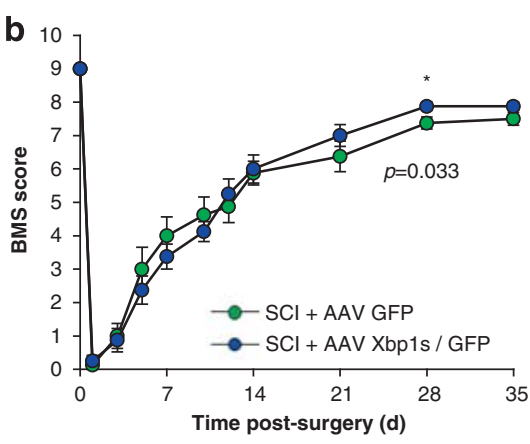

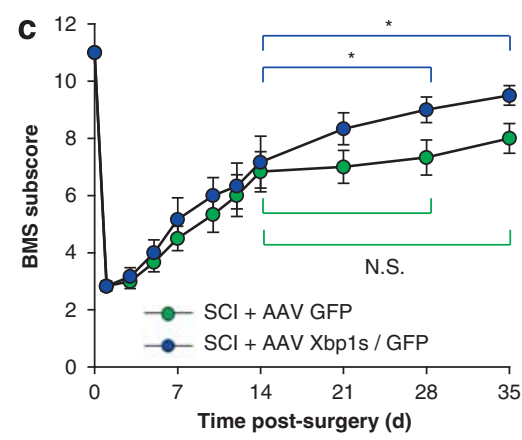

d

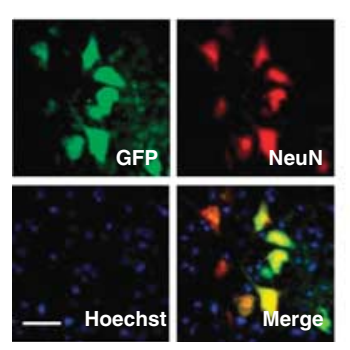

e

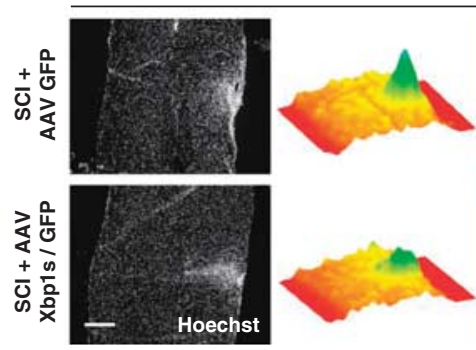

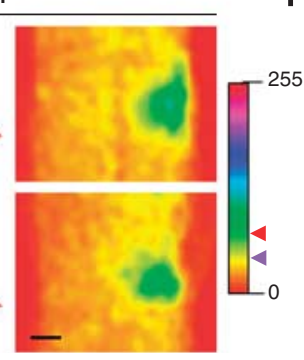

f Olig2 distribution

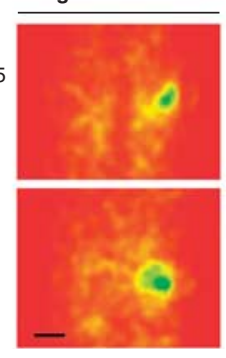

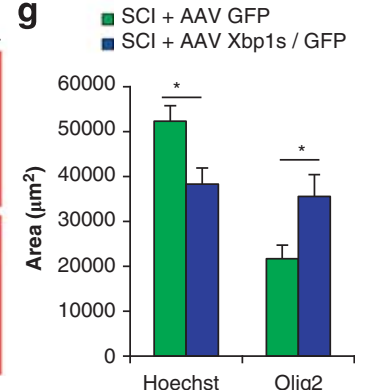

Figure 5 Xbp1s gene transfer with AAVs enhances locomotion recovery after SCl. Wild-type mice were hemisected at the T12 vertebral level and immediately injected into the injury site with $2 \mu \mathrm{l}\left(10^{12} \mathrm{DRP} / \mathrm{ml}\right)$ of AAV-GFP or AAV-Xbp1s/GFP. (a) Transduction levels were analyzed 35 days after injection by GFP expression (green), immunolabelled with an Olig2 antibody (red) and counterstained with Hoechst (blue) in the AAV-Xbp1s/GFP injections. (b) Locomotion recovery pattern was monitored before ( 0 day), and after spinal cord hemisection and viral transduction with AAV-GFP or AAV Xbp1s/GFP, using the BMS open-field test. (c) In the same groups, the BMS subscore was quantified to assess locomotor recovery of finer movements. (d) Neuronal-specific GFP transduction was confirmed by NeuN immunostaining (red) of spinal cord tissue injected with AAV Xbp1s/GFP (in green); nuclei were counterstained using Hoechst (blue). (e) Nuclear density in the injured region was analyzed by averaging Hoechst-positive nuclei intensity from eight mice for each condition and displayed as a surface plot. The Z axis represents average intensity in a pseudo-colored map. (f) Using the same approach as in (e), Olig2-positive cell density was studied in AAV-GFP or AAV Xbp1s/GFP-injected mice. (g) To quantitatively compare cellular density profiles, intensity plots of Hoechst- or Olig2-stained spinal cords from (e) and (f) were threshold at a fixed level (red arrowhead for Hoechst and purple arrowhead for Olig2 in the color map shown in (e)), and the resulting positive area was measured and plotted. Mean \pm S.E.M. ${ }^{\star} P<0.05$. Two-way repeated-measures ANOVA followed by Bonferroni's post hoc test for panels (b) and (c); $n=8$ animals per group. Student's $t$-test for panel $(\mathbf{g}) ; n=8$ animals per group. Scale bars, $300 \mu \mathrm{m}$ in $(\mathbf{a}), 20 \mu \mathrm{m}$ in $(\mathbf{d})$, and $300 \mu \mathrm{m}$ in (e) and (f)

to (i) restore the locomotor capacity of the affected individual or to (ii) attenuate the pathological events associated with spinal cord damage. Recent studies indicate that ER stress is an early tissue reaction after $\mathrm{SCl}$ in different models, including mechanical trauma and hypoxia..$^{8,10-13,27}$ Here we show a fast activation of a range of UPR responses after $\mathrm{SCI}$, including the expression of the proximal components ATF4 and XBP1, major transcription factors governing this pathway, in addition to the upregulation of downstream target genes. Remarkably, UPR activation was also observed in regions distant from the primary injury zone, suggesting the activation of a broad ER stress response in the spinal cord after damage. Interestingly, a recent report in cancer models suggested that under ER stress conditions, a transmissible signal related to proinflammatory events propagates the protein-folding stress response to the surrounding tissue, ${ }^{28}$ a phenomena that may also operate after SCl. Altered axonal trafficking due to spinal cord hemisection may also contribute to the activation of the UPR at distal regions because of a traffic jam in vesicular transport. We also analyzed available microarray data sets of $\mathrm{SCl}$-contusion models in mice (Gene Expression Omnibus, $\mathrm{NCBI}$ ) that confirmed a strong induction of sustained ER stress responses (Supplementary Figure S4).

Using genetic manipulation of the UPR, we demonstrated a functional role of this stress pathway in locomotor recovery after SCI. A recent study described the contribution of the proapoptotic transcription factor $\mathrm{CHOP}$ in models of mild SCI. ${ }^{13}$ $\mathrm{CHOP}$ deficiency enhanced motor recovery after $\mathrm{SCl}$, correlating with enhanced content of oligodendrocyte and white-matter sparing. ${ }^{13} \mathrm{CHOP}$ is one of a large group of downstream targets of proximal UPR transcription factors that controls the expression of many late-phase genes related to apoptosis and protein synthesis. ${ }^{5}$ In contrast, in models of severe $\mathrm{SCl}$, ablation of chop did not have an effect on motor recovery. ${ }^{15}$ CHOP expression is mostly controlled by the elF2 $\alpha /$ ATF 4 signaling branch, which is activated not only by the UPR sensor PERK but also by the kinases GCN2, HRI, and $\mathrm{PKR}^{2}$ that are non-ER stress-dependent. Therefore, our genetic studies with XBP1-deficient animals, and the development of an AAV-XBP1s gene therapy approach, represent the first direct proof for an involvement of the ER stress in SCI.

We speculate that the UPR may enhance neuronal plasticity events and functional recovery during the recovery phase after $\mathrm{SCl}$ related to neuronal functionality (cell autonomous events). Axonal degeneration was enhanced in atf $4^{-1-}$ mice, correlating with impaired motor recovery after experimental $\mathrm{SCl}$. Axonal degeneration might be an indicator of oligodendrocyte dysfunction, having a direct impact on locomotion recovery. Consistent with these findings, the accumulation of oligodendrocytes near the injury zone was 
reduced after deleting atf4 and enhanced after overexpressing XBP1s. Cell-type-dependent ER stress responses have been previously observed in $\mathrm{SCl}$ models. ${ }^{10,12,13}$ It has been described that the presence of NG2-positive cells in the injury zone is associated with the stabilization and regeneration of dystrophic axons after $\mathrm{SCl}^{29}$ In addition, increased oligodendrocyte number may also enhance the functional recovery of spared axons that were not directly ruptured and altered by the pro-inflammatory/oxidative environment. Remarkably, Keirstead et al. ${ }^{30}$ reported a functional improvement of locomotion when human patients were transplanted with human embryonic cell-derived oligodendrocyte progenitor cells. We also observed differential microglial activation 5 days after injury in atf $4^{-/-}$mice, which may contribute to the attenuated motor recovery in these mice after SCl. Interestingly, activated microglia has been suggested to attenuate oligodendrocyte precursor cell proliferation in vitro, ${ }^{31}$ providing a possible link between both cell type responses following SCl. The differences in cytokine levels observed in atf $^{-/-}$mice could alter different cellular processes including proliferation, differentiation, and cell death associated with $\mathrm{SCl}^{29}$ On the basis of our observations, we speculate that the UPR may be involved in controlling oligodendrocyte-dependent protective functions in the injury site that could contribute to locomotion recovery after $\mathrm{SCl}$.

ER stress is proposed to be a relevant factor in the pathogenesis of many neurodegenerative diseases involving abnormal protein folding. ${ }^{1}$ We have previously investigated the impact of the UPR in neurodegenerative diseases. Ablation of XBP1 in a model of amyotrophic lateral sclerosis (ALS) has a contrasting effect on spinal cord from the results presented here in $\mathrm{SCl}$ models. Despite expectations that XBP1 deficiency would enhance the pathogenesis of mutant SOD1-mediated ALS, a dramatic neuroprotection was observed owing to an enhanced clearance of mutant SOD1 aggregates by macroautophagy. ${ }^{32}$ In sharp contrast, PERK haplo-insufficiency enhances ALS progression. ${ }^{33}$ Besides, XBP1 deficiency did not affect prion misfolding or pathogenesis in vivo. ${ }^{23}$ Other reports indicate that XBP1 has a protective effect in animal models of Parkinson's disease, Alzheimer's disease, and autosomal dominant retinitis pigmentosa. ${ }^{1}$ ATF4 deficiency results in resistance to oxidative stress in models of brain ischemia. ${ }^{34,35}$ PERK/elF2 $\alpha$ signaling has protective effects on axonal remyelination in models of experimental autoimmune encephalomyelitis, possibly because of the control of oligodendrocyte viability and function. $^{33}$ Together with the current study, these reports suggest an intriguing scenario where specific UPR signaling branches may have distinct and contrasting consequence depending on the nature of the pathological stimuli.

A variety of mechanisms underlying modifications of ER homeostasis may take place in specific disease contexts, and could include inhibition of ER-associated degradation, altered vesicular trafficking, and altered protein-folding networks among others. ${ }^{1}$ In addition, alterations in lipid, cholesterol, or calcium metabolism may also affect ER function in many diseases affecting the nervous system. Interestingly, the physiological role of the UPR has been mostly attributed to maintaining an efficient rate of protein synthesis and secretion in specialized secretory cells including B lymphocytes, pancreatic $\beta$ cells, salivary glands, and many others. ${ }^{5}$ Neuronal and glial populations with higher secretory requirements might display increased sensitivity to genetic and environmental factors that disrupt ER function. In this context, oligodendrocytes and neuropeptide-secretory neurons are of particular relevance for future studies in order to uncover the mechanisms underlying the impact of the UPR in SCI. In fact, correlative studies suggest that the protection of valproate $^{11,36}$ or sodium 4-phenylbutyrate ${ }^{14}$ against $\mathrm{SCI}$ models is associated with attenuated levels of ER stress markers. The partial improvement obtained after XBP1s gene transfer over a wild-type condition where the UPR is already activated suggests that locomotor improvements can be achieved with treatments of days after damage (considering the delay of $>1$ week for gene expression after AAV gene transfer). The improvements in locomotor recovery observed in the gene therapy experiments may be actually important in the context of a patient that could progress from a state of full paralysis to gaining partial control of some movements, having significant impact on the quality of life. Overall, this study together with recent findings, identifies the UPR pathway as a potential target to treat $\mathrm{SCl}$. Importantly, the use of small molecules or gene therapy strategies to attenuate ER stress or artificially engage UPR responses may have a beneficial impact to attenuate tissue damage and enhance locomotor recovery in conditions affecting the function of the spinal cord.

\section{Materials and Methods}

Experimental animals and surgical procedures. Animals of 8 weeks of age, with body weight between 20 and $25 \mathrm{~g}$ were used in this study. ATF4 knockout mice and XBP-1 conditional knockout mice were previously described. ${ }^{23,37}$ All animals were used on a pure $\mathrm{C} 57 \mathrm{bl} 6$ genetic background. Mice were anesthetized with a single dose of $330 \mathrm{mg} / \mathrm{kg}$ of 2-2-2 Tribromoethanol (Sigma, St. Louis, MO, USA) intraperitoneally. Then, the dorsal zone of the spinal cord was incised along the midline; the T12 vertebra was laminectomized to expose the spinal cord. At this level, the spinal cord was hemitransected on the right side using a vannas micro scissor (RS-5658, ROBOZ, Gaithersburg, MD, USA). Sham animals include the complete surgical procedure without hemitransection of the spine. During recovery, mice were placed in a temperature-controlled chamber. At different days after surgery, animals were euthanized by an overdose of anesthesia. Surgical interventions and animal care follows the Institutional Review Board's Animal Care of the University of Chile (CBA \# 0305 FMUCH).

Western blot analysis of spinal cord extracts. A 5-mm spinal cord tissue containing the hemitransected region (medial), in addition to rostral and caudal regions of the same size, were collected and homogenized in $0.1 \mathrm{M}$ phosphate buffered saline $(\mathrm{pH} 7.4)$ containing a protease inhibitor cocktail (PIC, Roche, Basel, Switzerland). Half of the homogenized volume was used for protein extraction and the other half was used for RNA extraction (see below). The first volume was re-homogenized by sonication in RIPA buffer $(20 \mathrm{mM}$ Tris at $\mathrm{pH} 8.0$, $150 \mathrm{mM} \mathrm{NaCl}, 0.1 \%$ SDS, $0.5 \%$ deoxycholate, and $0.5 \%$ Triton X-100) plus PIC and then samples were analyzed by SDS-PAGE. The following antibodies and dilutions were used; anti-HSP90, 1:5000 (sc-7947, H114, Santa Cruz, Santa Cruz, CA, USA), anti-ATF4, 1: 3000 (sc-200, C-20, Santa Cruz), anti-BIM, $1: 2000$ (sc-8267, M-20, Santa Cruz), and anti-ubiquitin 1: 1000 (sc-8017, P4D1, Santa Cruz).

RNA extraction and RT-PCR. Total RNA was prepared from spinal cord tissue previously homogenized in saline phosphate buffer (PBS) using TRIzol (Invitrogen, Carlsbad, CA, USA). cDNA was synthesized by iScript cDNA Synthesis (Bio-Rad, Hercules, CA, USA) using random primers $p(d N) 6$ (Roche, Basel, Switzerland). Quantitative real-time PCR was performed in an ABI PRISM7700 system (Applied Biosystems, Foster City, CA, USA) employing SYBRgreen fluorescent reagent (Applied Biosystems) using the following primers: bip forward 5'-TCATCGGACGCACTTGGAA-3'; bip reverse 5'-CAACCACCTTGAATGGC 
AAGA-3'; chop forward 5'-TGGAGAGCGAGGGCTTTG-3'; chop reverse 5'-GT CCCTAGCTTGGCTGACAGA-3'; actin forward $5^{\prime}$-CTCAGGAGGAGCAATGATC TTGAT- $3^{\prime}$; actin reverse $5^{\prime}$-TACCACCATGTACCCAGGCA-3'; IL-1 $\beta$ forward $5^{\prime}$-CA ACCAACAAGTGATATTCTCCATG-3'; IL-1 $\beta$ reverse $5^{\prime}$-GATCCACACTCTCCAG CTGCA-3'; IL-6 forward 5'-GAGGATACCACTCCCAACAGACC-3'; IL-6 reverse, $5^{\prime}$-AAGTGCATCATCGTTGTTCATACA-3'; and TNF $\alpha$ forward $5^{\prime}$-CATCTTCTCAAA ATTCGAGTGACAA-3'; TNF $\alpha$ reverse 5'-TGGGAGTAGACAAGGTACAACCC-3'. XBP1 mRNA splicing assay was performed as previously described using Pstl digestion of PCR products using the following primers: mXBP1.3S (5'-AAA CAGAGTAGCAGCGCAGACTGC-3') and mXBP1.2AS: (5'-GGATCTCTAAAACTAG AGGCTTGGTG-3').

Locomotor function. Locomotor recovery was evaluated in an open-field test using the nine-point Basso Mouse Scale (BMS). ${ }^{21}$ The BMS analysis of hindlimb movements and coordination for lateral hemisection $\mathrm{SCl}$ model was performed by two independent investigators blinded to the experimental condition as described before. ${ }^{38}$ Our laboratory was certified during the 2009 Spinal Cord Injury Research Training Program at Ohio State University, USA, to perform BMS assays.

BMS analysis involved measuring the following parameters and scores: (0) no ankle movement; (1) slight ankle movement; (2) extensive ankle movement; (3) plantar placing of the paw with or without weight support, or occasional, frequent or consistent dorsal stepping but no plantar stepping; (4) occasional plantar stepping; (5) frequent or consistent plantar stepping, no coordination or frequent, or consistent plantar stepping, some coordination, paws rotated at initial contact and lift off; (6) frequent or consistent plantar stepping, some coordination, paws parallel at initial contact, or frequent or consistent plantar stepping, mostly coordinated, paws rotated at initial contact and lift off; (7) frequent or consistent plantar stepping, mostly coordinated, paws parallel at initial contact and rotated at lift off, or frequent or consistent plantar stepping, mostly coordinated, paws parallel at initial contact and lift off, and severe trunk instability; (8) frequent or consistent plantar stepping, mostly coordinated, paws parallel at initial contact and lift off, and mild trunk instability, or frequent or consistent plantar stepping, mostly coordinated, paws parallel at initial contact and lift off, and normal trunk stability, and tail down or up and down; (9) frequent or consistent plantar stepping, mostly coordinated, paws parallel at initial contact and lift off, and normal trunk stability and tail always up. In general, the score for individual hindlimb ranged from 0 to 5 points (parameters that do not involve coordination); and parameters involving the use of both hind limbs reached between 6 and 9 points. The 11-point BMS subscore was used to evaluate finer aspects of locomotor capacity, which are not revealed by the BMS. ${ }^{21}$ BMS subscore included the following interval of scores: plantar stepping (0-2); coordination (0-2); paw position (0-4); trunk stability (0-2); and tail position (0-1).

To control the surgery, the following criteria of exclusion were employed: (i) at 1 day after surgery, mice with $<5$ points in its contralateral hindlimb were excluded for the study (around 1 of 16 animals). (ii) If the BMS score for the ipsilateral hindlimb was $>1$ point at 1 day after surgery, the mouse was excluded for the study (around 1 of 16 animals). The final score is presented as mean \pm S.E.M.

Histological analysis. At 5 or 14 days after surgery, mice were perfused transcardially with $4 \%$ paraformaldehyde in $0.1 \mathrm{M} \mathrm{PBS}$. A $5-\mathrm{mm}$ region of the spinal cord containing the lesion site was removed and post-fixed for $3 \mathrm{~h}$ in $4 \%$ paraformaldehyde. The spinal tissue was subjected to a sucrose gradient $(5,10$, and $30 \%$ sucrose in PBS), cryoprotected with optimal cutting temperature compound (Tissue-Tek, Alphen aan den Rijn, The Netherlands), and fast frozen using liquid nitrogen. The tissue was longitudinally sectioned (5 $\mu$ m-thick slices) using a cryostat microtome (Leica, Nussloch, Germany). Sections were immunostained using antibodies anti-Cd11b, 1:100 (MCA74G, Serotec, Morphosys, Oxford, UK), NeuN 1:300 (MAB377, Millipore Bioscience Research Reagents, Billerica, MA, USA), anti-Olig-2 1:200 (ab9610, Millipore Bioscience Research Reagents), and GFAP 1:1000 (N1506, Dako, Glostrup, Denmark). Tissue sections were viewed with an Olympus IX71 microscope (Olympus, Center Valley, PA, USA) and images were captured using a QImaging QICAM Fast 1394 camera (Surrey, BC, Canada). Olig2-positive cells surrounding the injury zone were analyzed using a matrix of $50 \mu \mathrm{m}$-separated concentric semicircles. GFAP staining intensity was calculated by creating an integrated intensity profile along the spinal cord with its center located in the injury site. Neuronal numbers were assessed by counting NeuN-positive cells in the spinal tissue, excluding the mechanically injured region. Cd11b-positive cells were determined in the region surrounding the mechanically injured zone. Longitudinal SC slices were also used for eriochrome/ cyanine staining. Slices were imaged using an OLYMPUS CX31 microscope fitted with a CCD camera. All quantifications were done using ImageJ software $(\mathrm{NIH}$, Bethesda, MD, USA). For EM analyses, spinal cord tissue was processed as described. ${ }^{39}$ Resin slices measuring $1 \mu \mathrm{m}$ were cut and toluidine blue stained as described. ${ }^{39}$ Slices were imaged using an Olympus IX71 microscope fitted with a QImaging QICAM Fast 1394 camera.

AAV production and injection of viral particles into the spinal cord. The whole Xbp-1 expression cassette was excised from pcDNA3XBP-1S as a Mfel/Sphl fragment and inserted into a proviral plasmid pAAVsp70 containing AAV2-inverted terminal repeats (ITRs). The vector is bicistronic and carries a GFP expression cassette that serves as a fluorescent marker for transduced cells. Recombinant AAV2.XBP1S was produced by triple transfection of 293 cells using a rep/cap plasmid and pHelper (Stratagene, La Jolla, CA, USA) and purified by column affinity chromatography, as previously described. ${ }^{40}$ Viral titers were determined using a real-time TaqMan PCR assay (ABI Prism 7700; Applied Biosystems) with primers that were specific for the BGH polyA sequence. For animal treatment, $2 \mu \mathrm{l}$ of AAVs $\left(10^{12} \mathrm{DRP} / \mathrm{ml}\right)$ were injected slowly over the injury site, right after performing the hemisection using a 10- $\mu$ l Hamilton syringe fitted with a $34 \mathrm{G}$ needle. We confirmed the expression of XBP1s protein and upregulation of the target genes EDEM1, ERp72, and Sec61 after the stereotaxis injection of AAV-XBP1 into the brain followed by real time PCR analysis (not shown).

Statistical analysis. Data are shown as mean \pm S.E.M. Statistical analyses were performed by using Student's $t$ test or two-way repeated-measures ANOVA, followed by a Bonferroni post hoc test for multiple comparisons.

\section{Conflict of Interest}

The authors declare no conflict of interest.

Acknowledgements. We thank Monica Perez for excellent EM processing and Alejandra Catenaccio for technical assistance. This work was primarily supported by a North American Spine Society (NASS) Award. We also thank for the financial support from FONDECYT No. 1100176, FONDAP No. 15010006, Millennium Institute No. P09-015-F, Alzheimer's Association, Muscular Dystrophy Association, The Michael J. Fox Foundation for Parkinson's Research, and ICGEB (to CH). CONICYT Doctoral fellowship (EC) and FONDECYT No. 1110987, Millennium Nucleus No. P07-011-F (FC).

1. Matus S, Glimcher LH, Hetz C. Protein folding stress in neurodegenerative diseases: a glimpse into the ER. Curr Opin Cell Biol 2011; 23: 239-252.

2. Hetz $C$. The unfolded protein response: controlling cell fate decisions under ER stress and beyond. Nat Rev Mol Cell Biol 2012; 13: 1-14.

3. Woehlbier U, Hetz $\mathrm{C}$. Modulating stress responses by the UPRosome: a matter of life and death. Trends Biochem Sci 2011; 36: 329-337.

4. Ron D, Walter $P$. Signal integration in the endoplasmic reticulum unfolded protein response. Nat Rev Mol Cell Biol 2007; 8: 519-529.

5. Hetz C, Martinon F, Rodriguez D, Glimcher LH. The unfolded protein response: integrating stress signals through the stress sensor IRE1 $\alpha$. Physiol Rev 2011; 91: 1219-1243.

6. Harding HP, Zhang Y, Ron D. Protein translation and folding are coupled by an endoplasmic-reticulum-resident kinase. Nature 1999; 397: 271-274.

7. Schroder M, Kaufman RJ. The mammalian unfolded protein response. Annu Rev Biochem 2005; 74: 739-789.

8. Aufenberg C, Wenkel S, Mautes A, Paschen W. Spinal cord trauma activates processing of xbp1 mRNA indicative of endoplasmic reticulum dysfunction. J Neurotrauma 2005; 22: 1018-1024.

9. Paschen W, Aufenberg C, Hotop S, Mengesdorf T. Transient cerebral ischemia activates processing of xbp1 messenger RNA indicative of endoplasmic reticulum stress. Blood 2003; 23: 449-461.

10. Penas C, Guzman MS, Verdu E, Fores J, Navarro X, Casas C. Spinal cord injury induces endoplasmic reticulum stress with different cell-type dependent response. $J$ Neurochem 2007; 102: 1242-1255.

11. Penas C, Verdú E, Asensio E, Guzmán-Lenis MS, Herrando-Grabulosa M, Navarro X et al. Valproate reduces $\mathrm{CHOP}$ levels and preserves oligodendrocytes and axons after spinal cord injury. Neuroscience 2011; 178: 1-12.

12. Yamauchi $T$, Sakurai $M, A$ be $K$, Matsumiya $G$, Sawa $Y$. Impact of the endoplasmic reticulum stress response in spinal cord after transient ischemia. Brain Res 2007; 1169: 24-33.

13. Ohri SS, Maddie MA, Zhao Y, Qiu MS, Hetman M, Whittemore SR. Attenuating the endoplasmic reticulum stress response improves functional recovery after spinal cord injury. Glia 2011; 59: 1489-1502. 
14. Mizukami T, Orihashi K, Herlambang B, Takahashi S, Hamaishi M, Okada K et al. Sodium 4-phenylbutyrate protects against spinal cord ischemia by inhibition of endoplasmic reticulum stress. J Vasc Surg 2010; 52: 1580-1586.

15. Ohri SS, Maddie MA, Zhang YP, Shields CB, Hetman M, Whittemore SR. Deletion of the pro-apoptotic endoplasmic reticulum stress response effector CHOP does not result in improved locomotor function after severe contusive spinal cord injury. J Neurotrauma 2011; e-pub ahead of print 21 November 2011.

16. Luethy JD, Holbrook NJ. Activation of the gadd153 promoter by genotoxic agents: a rapid and specific response to DNA damage. Cancer Res 1992; 52: 5-10.

17. Tang JR, Nakamura M, Okura T, Takata Y, Watanabe S, Yang ZH et al. Mechanism of oxidative stress-induced GADD153 gene expression in vascular smooth muscle cells. Biochem Biophys Res Comm 2002; 290: 1255-1259.

18. Hotamisligil GS. Endoplasmic reticulum stress and the inflammatory basis of metabolic disease. Cell 2010; 140: 900-917.

19. Ameri K, Harris AL. Activating transcription factor 4. Int J Biochem Cell Biol 2008; 40: $14-21$

20. Puthalakath $\mathrm{H}$, O'reilly LA, Gunn P, Lee L, Kelly PN, Huntington ND et al. ER stress triggers apoptosis by activating BH3-only protein Bim. Cell 2007; 129: 1337-1349.

21. Basso DM, Fisher LC, Anderson AJ, Jakeman LB, McTigue DM, Popovich PG. Basso mouse scale for locomotion detects differences in recovery after spinal cord injury in five common mouse strains. J Neurotrauma 2006; 23: 635-659.

22. Hetz C, Glimcher LH. Fine-tuning of the unfolded protein response: assembling the IRE1a interactome. Mol Cell 2009; 34: 1-11.

23. Hetz C, Lee AH, Gonzalez-Romero D, Thielen P, Castilla J, Soto C et al. Unfolded protein response transcription factor XBP-1 does not influence prion replication or pathogenesis. Proc Natl Acad Sci USA 2008; 105: 757-762.

24. Raasch J, Zeller N, van Loo G, Merkler D, Mildner A, Erny D et al. IkappaB kinase 2 determines oligodendrocyte loss by non-cell-autonomous activation of NF-kappaB in the central nervous system. Brain 2011; 134 (Pt 4): 1184-1198.

25. Davidson BL, Stein CS, Heth JA, Martins I, Kotin RM, Derksen TA et al. Recombinan adeno-associated virus type 2, 4, and 5 vectors: transduction of variant cell types and regions in the mammalian central nervous system. Proc Natl Acad Sci USA 2000; 97: 3428-3432.

26. Zincarelli C, Soltys S, Rengo G, Rabinowitz JE. Analysis of AAV serotypes 1-9 mediated gene expression and tropism in mice after systemic injection. Mol Ther 2008; 16: 1073-1080.

27. Hong Z, Hong $\mathrm{H}$, Chen $\mathrm{H}$, Wang $\mathrm{Z}$, Hong D. Protective effects of erythropoietin in experimental spinal cord injury by reducing the C/EBP-homologous protein expression. Neurol Res 2012; 34: 85-90.

28. Mahadevan NR, Rodvold J, Sepulveda H, Rossi S, Drew AF, Zanetti M. Transmission of endoplasmic reticulum stress and pro-inflammation from tumor cells to myeloid cells. Proc Natl Acad Sci U S A 2011; 108: 1-6.
29. Lees JR, Cross AH. A little stress is good: IFN-gamma, demyelination, and multiple sclerosis. J Clin Invest 2007; 117: 297-299.

30. Keirstead HS, Nistor G, Bernal G, Totoiu M, Cloutier F, Sharp K et al. Human embryonic stem cell-derived oligodendrocyte progenitor cell transplants remyelinate and restore locomotion after spinal cord injury. J Neurosci 2005; 25: 4694-4705.

31. Taylor DL, Pirianov G, Holland S, McGinnity CJ, Norman AL, Reali C et al. Attenuation of proliferation in oligodendrocyte precursor cells by activated microglia. J Neurosci Res 2010; 88: $1632-1644$.

32. Hetz C, Thielen P, Matus S, Nassif M, Court F, Kiffin R et al. XBP-1 deficiency in the nervous system protects against amyotrophic lateral sclerosis by increasing autophagy. Genes Dev 2009; 23: 2294-2306.

33. Wang L, Popko B, Roos RP. The unfolded protein response in familial amyotrophic lateral sclerosis. Hum Mol Genet 2011; 20: 1008-1015.

34. Lange PS, Chavez JC, Pinto JT, Coppola G, Sun CW, Townes TM et al. ATF4 is an oxidative stress-inducible, prodeath transcription factor in neurons in vitro and in vivo. J Exp Med 2008; 205: 1227-1242.

35. Galehdar Z, Swan P, Fuerth B, Callaghan SM, Park DS, Cregan SP. Neuronal apoptosis induced by endoplasmic reticulum stress is regulated by ATF4-CHOP-mediated induction of the Bcl-2 homology 3-only member PUMA. J Neurosci 2010; 30: 16938-16948.

36. Ryoo HD, Domingos PM, Kang MJ, Steller H. Unfolded protein response in a Drosophila model for retinal degeneration. Embo J 2007; 26: 242-252.

37. Tanaka T, Tsujimura T, Takeda K, Sugihara A, Maekawa A, Terada N et al. Targeted disruption of ATF4 discloses its essential role in the formation of eye lens fibres. Genes Cells 1998; 3: 801-810.

38. Boido M, Rupa R, Garbossa D, Fontanella M, Ducati A, Vercelli A. Embryonic and adult stem cells promote raphespinal axon outgrowth and improve functional outcome following spinal hemisection in mice. Eur J Neurosci 2009; 30: 833-846.

39. Barrientos SA, Martinez NW, Yoo S, Jara JS, Zamorano S, Hetz C et al. Axonal degeneration is mediated by the mitochondrial permeability transition pore. $J$ Neurosci 2011; 31: 966-978.

40. O'Riordan CR, Lachapelle AL, Vincent KA, Wadsworth SC. Scaleable chromatographic purification process for recombinant adeno-associated virus (rAAV). J Gene Med 2000; 2: 444-454.

Cell Death and Disease is an open-access journal published by Nature Publishing Group. This work is licensed under the Creative Commons Attribution-Noncommercial-No Derivative Works 3.0 Unported License. To view a copy of this license, visit http://creativecommons.org/licenses/by-nc-nd/3.0/

\section{Supplementary Information accompanies the paper on Cell Death and Disease website (http://www.nature.com/cddis)}

